\title{
Diarrea intratable causada por anomalías congénitas de los enterocitos
}

\author{
Alan D. Phillips \\ Centro de Gastroenterología Pediátrica, Royal Free Hospital, Londres, Reino Unido
}

\section{Palabras clave}

Diarrea intractable $\cdot$ Lactancia $\cdot$ Enteropatía

\section{Extracto}

Fundamento: La diarrea intratable de la lactancia representa un problema grave que conlleva un grado elevado de morbilidad y mortalidad. Estos casos requieren una asistencia dedicada tanto por parte de los padres como del personal médico, dado que no se dispone de remedios fáciles. La escasez de pacientes indica que para progresar en el conocimiento de estos trastornos son necesarios esfuerzos internacionales concertados para combinar recursos y pacientes. Son indispensables revisiones regulares de publicaciones pertinentes para propagar los avances y airear hipótesis y criterios diagnósticos. Métodos: Ésta es una revisión de la literatura actual y preliminar sobre las anomalías congénitas de los enterocitos. Resultados: Es posible establecer diagnósticos separados dentro de este síndrome, y los criterios diagnósticos están empezando a ser aceptados. Se está informando de casos a nivel mundial y las diferencias en los cursos clínicos están empezando a evidenciarse, con mejoría de la gravedad en algunos casos. No obstante, persiste un nivel elevado de morbilidad y mortalidad. Conclusiones: Cabe recorrer todavía un largo trecho hasta poder determinar la base subyacente de los trastornos. No obstante, la esperanza de determinar la base genética de las enfermedades está empezando a ser una realidad a medida que se realicen análisis genéticos moleculares. Esto es prioritario si tenemos en cuenta la posibilidad de que proporcione criterios diagnósticos más evidentes, aclarare las relaciones de la enfermedad y suministre una información muy necesaria sobre la patogénesis.

Copyright (C) 2006 Nestec Ltd., Vevey/S. Karger AG, Basel

\section{Introducción}

La diarrea intratable sigue siendo un problema pediátrico grave con un elevado grado de morbilidad y mortalidad. Representa una carga individual espectacular capaz de desafiar al tratamiento. Exige un cuidado comprometido tanto por parte de los padres como del personal médico. Este cuadro particular de diarrea intratable, es decir, las anomalías congénitas de los enterocitos, afecta frecuentemente a lactantes recién nacidos en los primeros días de vida. Las complejidades en relación con el acceso tisular y venoso para el diagnóstico y el tratamiento empeoran significativamente los problemas que causan las pérdidas diarreicas, que pueden representar hasta el 10\% o más del peso corporal por día. No obstante, es posible establecer diagnósticos separados. La nutrición parenteral ha permitido una supervivencia a plazo relativamente largo, el trasplante intestinal ofrece esperanzas para

\section{KARGER}

Fax +4161306 1234 E-Mail karger@karger.ch www.karger.com
C) 2006 Nestec Ltd. Vevey/S. Karger AG, Basel 0252-8185/06/0641-0015\$23.50/0

Accesible online en: www.karger.com/ans
Alan D. Phillips, $\mathrm{PhD}$

Centre for Paediatric Gastroenterology

Lower 3rd Floor, Royal Free Hospital, Pond Street

London, NW3 2QG (UK)

Tel. +44 207830 2783, Fax +44 207830 2146, E-Mail a.phillips@medsch.ucl.ac.uk 
una vida sin patología y la genética molecular se está aplicando en un intento de aclarar las anomalías genéticas que, sin duda, reposan en los cimientos de la enfermedad.

El término diarrea intratable fue introducido por Avery et al. [1] a comienzos de los años 60. Estos autores estudiaron a un grupo de 20 niños menores de 3 meses con diarrea crónica (de duración superior a 14 días), coprocultivo negativo y diarrea persistente a pesar de las intervenciones médicas.

Todos ellos eran candidatos de nutrición parenteral y nueve fallecieron. Se publicaron trabajos similares en los años siguientes $[2,3]$ en los que se daba a entender que el proceso era hereditario, implicaba lesión intestinal y que, probablemente, la responsabilidad recaía en más de una sola entidad patológica (enteropatía familiar [4] y diarrea prolongada familiar letal [5]).

Davidson et al. [4] describieron la enteropatía como atrofia vellosa hipoplásica de las criptas sin inflamación de la lamina propria, destacando la falta de evidencia de hiperplasia de las criptas (tal como se observa en la celiaquía) y un componente de origen inmunitario. Si bien en estudios posteriores se puso en duda la naturaleza hipoplásica de la enteropatía (ver más adelante), esta observación siguió adelante y se aplicaron criterios inmunohistológicos a casos de diarrea intratable [6,7]. Este enfoque permitió una división entre enfermedades mediadas por el sistema inmunitario y enfermedades basadas en el epitelio, de manera que se aclararon las categorías de esta patología [7] y se propuso la optimación de las opciones terapéuticas [8]. En este artículo nos centraremos en las anomalías basadas en el epitelio y no consideraremos la enteropatía autoinmune y los trastornos u opciones terapéuticas relacionados, mediados por el sistema inmunitario, dado que se abordan en otra parte.

Tampoco se considera la enterocolitis ulcerosa idiopática de la lactancia $[9,10]$, dado que sus características son más similares a la diarrea intratable de origen inmunitario.

Hasta la fecha se ha identificado un escaso número de diagnósticos separados dentro del grupo de anomalías congénitas de los enterocitos asociadas a diarrea intratable. Aunque el nivel de evidencia para situarlas en esta categoría es variable, cuando menos se trata de una enteropatía y no, o apenas, de una inflamación de la lamina propria. Las anomalías subyacentes de estos procesos no se han establecido y se está investigando para determinar sus bases genéticas moleculares. Hasta el momento, no se han descubierto anomalías en los genes. Entre los diagnósticos destacan la atrofia de las microvellosidades o enfermedad de inclusión de microvellosidades, la enteropatía en penacho o displasia epitelial, la diarrea sindromática o síndrome trico-hepático-entérico, la enterocolitis ulcerosa idiopática de la lactancia y trastornos de la glucosilación, a cuyas entidades deben añadirse casos idiopáticos. Estos se abordarán en su momento, más adelante. Se han publicado varios artículos de revisión referentes a los diagnósticos más corrientes dentro de la diarrea intratable del síndrome de lactancia [7, 9-15], y el propósito de esta revisión es evitar una repetición excesiva.

\section{Atrofia de las microvellosidades/enfermedad de inclusión de microvellosidades}

En 1982, en la reunión anual del ESPGHAN, celebrada en Madrid, se presentaron dos extractos en los que se destacaron los signos raros detectados en el epitelio de lactantes con diarrea intratable. Schmitz et al. [16] acuñaron el término atrofia de las microvellosidades, mientras que Goutet et al. [17] remitían a Davidson et al. [4] con el término descriptivo, enteropatía familiar con atrofia vellosa hipoplásica. Ambos describían los signos de inclusiones de microvellosidades dentro del epitelio, que habían sido descritas ya por Davidson et al. [4] en una de sus series de 5 lactantes; además, informaron de la presencia de 'gránulos secretores' en células de las criptas y en enterocitos superficiales [4]. Nosotros publicamos otros 2 casos de diarrea intratable de origen congénito, que presentaban una enteropatía con las mismas características distintivas (es decir, inclusiones de microvellosidades e incremento de 'gránulos secretores'; fig. 1,2) y dejamos entrever que se trataban de características diagnósticas de la enfermedad [18]. Subsiguientemente, Cutz et al. [11] propusieron el término de enfermedad de inclusión de microvellosidades con objeto de resaltar la anomalía de estas últimas.

\section{Datos clínicos}

Los datos clínicos están bien descritos y se ha aceptado tanto su gravedad como que su inicio tiene lugar habitualmente en el curso de la primera semana de vida $[4,11$, $12,18]$. La diarrea persiste en ausencia de nutrición enteral y se exacerba con la nutrición oral [12]. Es evidente una distribución mundial de la enfermedad, con casos descritos en Europa [12], Oriente Medio [12], Asia [19, 20] y Norteamérica [11], incluyendo a norteamericanos nativos (tribu de los indios navajos) [21]. Aunque son pocos los casos descritos en África, el autor ha confirma- 

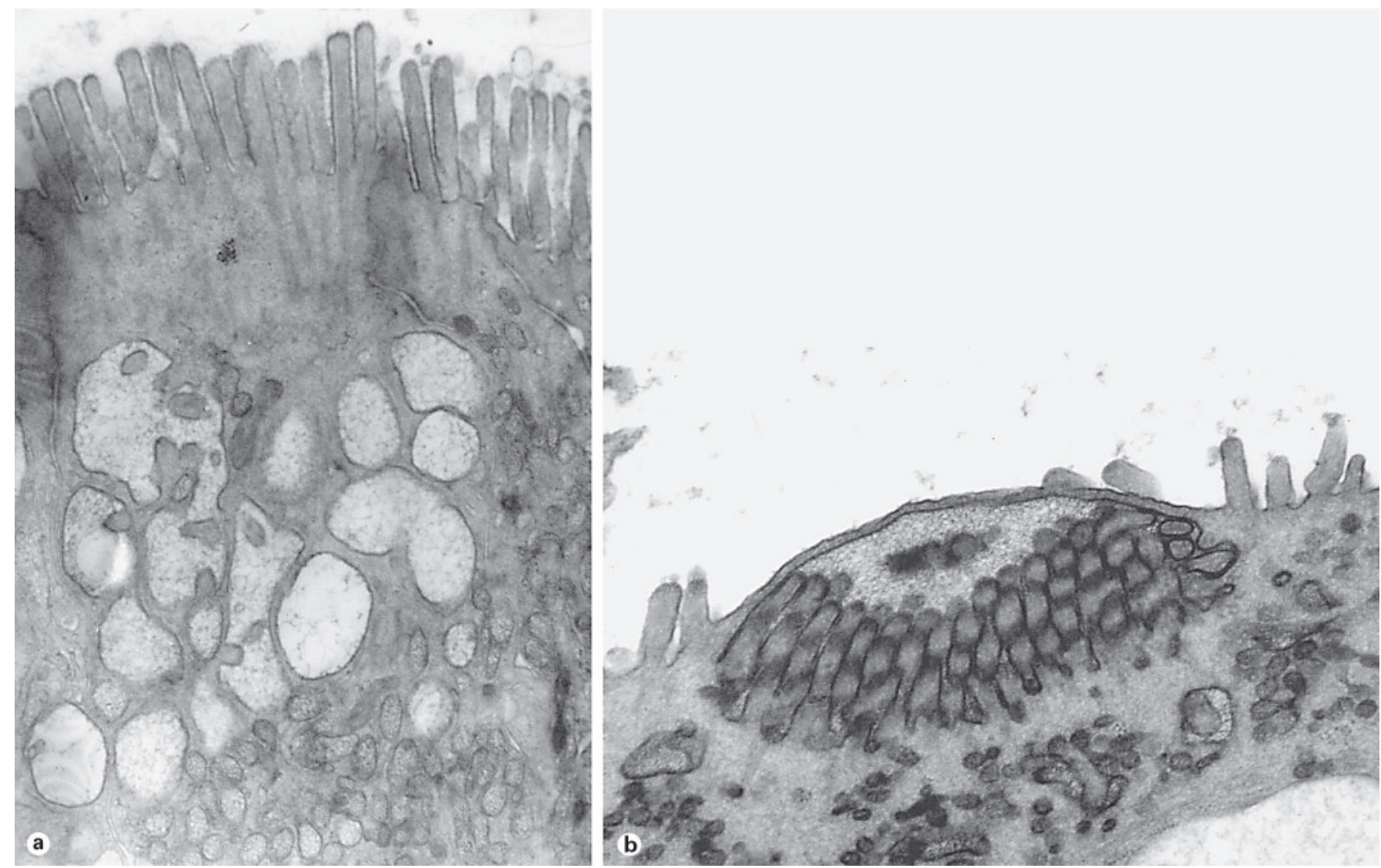

Fig. 1. Atrofia de las microvellosidades. a Desarrollo manifiesto de microvellosidades dentro de una célula con ribete en cepillo intacto, aunque corto. b Inclusión de microvellosidades de tipo vesicular con aspecto de envoltura.
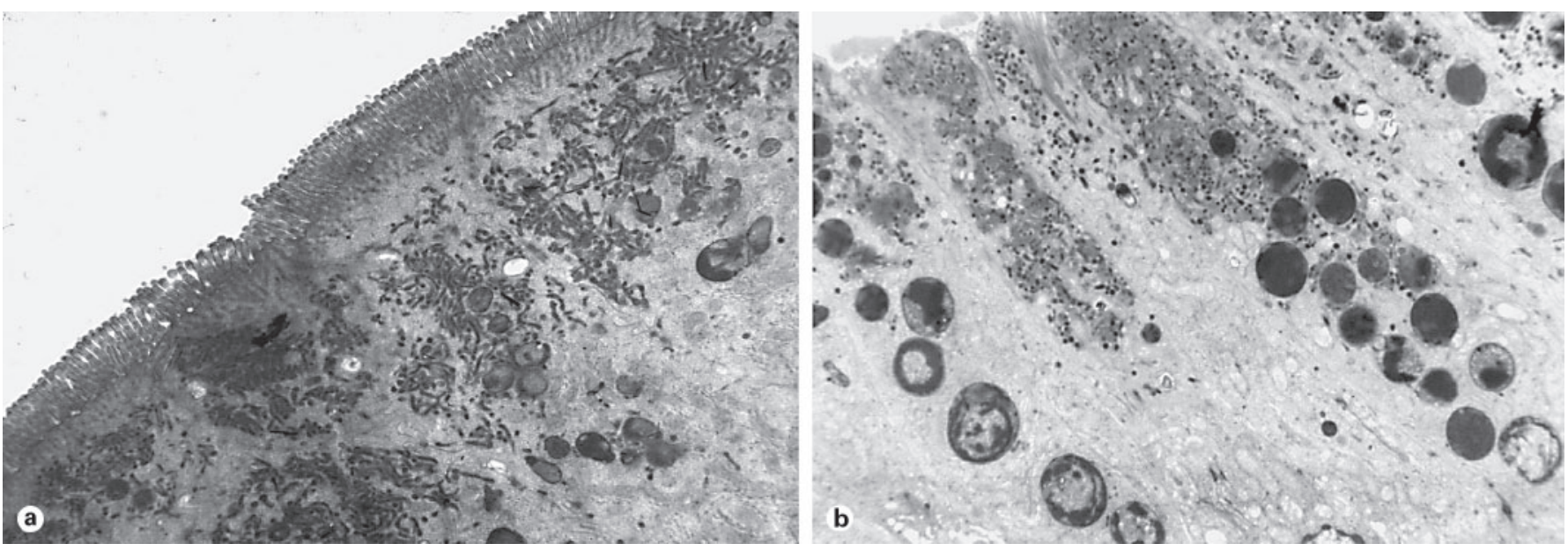

Fig. 2. Atrofia de las microvellosidades. Gránulos secretores de inicio tardío (a) y casos atípicos (b). 
do el diagnóstico de atrofia de las microvellosidades en un norteamericano de decendencia africana. En países en vías de desarrollo, la gravedad de la enfermedad puede impedir su diagnóstico.

Aunque la mayoría de los casos se presenta en la primera semana de vida, en algunos el inicio de los síntomas tiene lugar después del periodo neonatal; en estos casos se habla de atrofia de las microvellosidades de 'inicio tardío' [12]. Aparecen las características anomalías morfológicas intestinales aunque con diferencias tenues (ver más adelante), que dificultan el diagnóstico. Aunque los supervivientes a largo plazo afectos de la enfermedad son pocos, se considera que los casos de 'inicio tardío' pueden conllevar un mejor pronóstico [12]. Es demasiado pronto para aceptar esta posibilidad, aunque en una serie de 23 casos, 3 de 5 supervivientes eran de 'inicio tardío' [12]. El caso 9 de esta serie tiene actualmente 23 años de edad y evoluciona relativamente bien con nutrición parenteral parcial, aunque sin signos de mejoría de su anomalía intestinal [Ventura A., comunicación personal].

A medida que se han ido diagnosticando más casos se ha descrito una gama más amplia de modalidades de presentación y tratamiento. Son interesantes, dado que pueden aclarar la patogénesis subyacente y/o servir de recordatorios para considerar el diagnóstico en situaciones que no corresponden al dogma aceptado, si bien la mayoría de los casos se presenta tal como se describe en la literatura $[4,11,12,18]$. Aunque en la mayor parte de los casos no se asocia a polihidramnios materno, se describió recientemente un caso con polihidramnios y obstrucción intestinal antenatal evidente, que resultó en atrofia de las microvellosidades [22]. En consecuencia, la atrofia de las microvellosidades debe considerarse como parte del diagnóstico diferencial ante la posibilidad de una diarrea de cloruros [23] y una deficiencia en el intercambio Na-H [24]. Otro caso de 'inicio tardío' (3 meses) y signos diagnósticos característicos ganó peso espontáneamente a los 2 años de edad, a pesar de la persistencia de la diarrea; esta niña dejó de necesitar nutrición parenteral a los 4 años [25]. Si bien este caso representa un cuadro inusitado de esta enfermedad, el trasplante intestinal precoz [26] no hubiera sido apropiado para esta paciente y, tal vez, tampoco para otros casos de 'inicio tardío'.

Se observó otro acontecimiento interesante en un caso que permanecía a la espera de trasplante intestinal y hepático [27]. Tras una biopsia del hígado presentó una hemorragia intraperitoneal grave que obligó a una intervención quirúrgica; el shock hemorrágico de riesgo vital cursó con insuficiencia renal. Al mismo tiempo su diarrea disminuyó, dejó de evacuar deposiciones durante una semana y, seguidamente, las emisiones continuaron al ritmo de $10-30 \mathrm{ml} / \mathrm{kg} /$ día hasta el momento del trasplante. La nutrición enteral ocasionó un incremento de la evacuación de deposiciones y no se registraron cambios en la morfología intestinal. Una reducción extrema de la presión hidrostática indujo, aparentemente, un cambio en la naturaleza secretora de la diarrea.

La enfermedad obedece probablemente a un rasgo autosómico recesivo debido al número de padres consanguíneos y al hallazgo de familias con hermanos afectados $[12,28]$. Aunque se han descrito enfermedades genéticas coincidentes [29, 30], no se conoce a ciencia cierta si representan síndromes de genes contiguos o son heredadas independientemente. El elevado número de padres consanguíneos abre la senda de los estudios genéticos moleculares y la posibilidad de establecer la base genética de esta enfermedad.

Casos clínicamente similares de diarrea intratable sin el diagnóstico de características intestinales de atrofia de las microvellosidades alentaron la propuesta de que tales casos pueden estar relacionados y son indicativos de un espectro de trastornos de las microvellosidades $[31,32]$, que han recibido la denominación de distrofia de las microvellosidades intestinales [31]. Cabe la posibilidad de que los signos diagnósticos característicos hayan pasado inadvertidos [33], y el grado de similitud con la atrofia de las microvellosidades exige la realización de estudios ulteriores, incluyendo el análisis genético molecular.

\section{Patología}

Se trata de una enteropatía con atrofia de las vellosidades, pero sin hiperplasia intensa de las criptas, que genera una mucosa fina [12]; no hay inflamación de la lamina propria. No se trata de una atrofia hipoplásica de las vellosidades [4], dado el incremento del número de células de las criptas [12, 34], así como de la apoptosis y la proliferación [35]. La ausencia de hiperplasia de las células de las criptas no se debe a una capacidad limitada de las criptas para responder, dado que el tratamiento con factor de crecimiento epidérmico incrementa la proliferación de las células intestinales pero no mejora el estado clínico [34]. La muerte de células epiteliales daña la digestión y la absorción y es uno de los motivos de la situación diarreica.

Si el intestino delgado se tiñe con tinción de ácido peryódico de Schiff (APS) se observa una acumulación anormal de material APS-positivo en el citoplasma apical de las células epiteliales. En la atrofia de las microvellosi- 
dades congénita este cuadro aparece en el epitelio superior de las criptas, mientras que en los casos de 'inicio tardío' la acumulación aparece en el epitelio bajo de las vellosidades y se observa una tinción APS normal en el ribete en cepillo de las células inferiores [12]. En una tercera variante, denominada atrofia de las microvellosidades 'atípica', la anomalía APS aparece en la parte inferior de las criptas, y hacia el epitelio superficial se observa cierta tinción APS normal en el ribete en cepillo [12]. La anomalía APS permite suponer que una sustancia destinada al ribete en cepillo se acumula dentro de la célula en lugar de ser trasportada a la membrana apical. Esta distribución discurre paralelamente a la observación por microscopía electrónica (ME) de un incremento de los 'gránulos secretores'. Es probable que ambas anomalías representen un mismo fenómeno [36]. Por lo tanto, los gránulos secretores contienen material que se sitúa normalmente en el ribete en cepillo. La ME de los 'gránulos' demostró la ausencia de gránulos separados y la constitución de una red vesicular [36]. El complejo de Golgi era normal, lo que indica un bloqueo post-Golgi de la exocitosis. El APS sistemático confiere la oportunidad de establecer un diagnóstico retrospectivo [36]; la ME ha demostrado el otro distintivo de la atrofia de las microvellosidades, a saber, la presencia de vacuolas revestidas de microvellosidades, o inclusiones, dentro del citoplasma apical de las células epiteliales superficiales (fig. 1); es decir, que aparecen en un momento más tardío del ciclo vital de las células epiteliales que los gránulos secretores. Pueden aparecer como inclusiones pequeñas o grandes y, en ocasiones, se presentan como invaginaciones de la membrana apical o como configuraciones 'de tipo vesicular' con microvellosidades en uno de los lados de la inclusión (fig. 1b). Las inclusiones de microvellosidades experimentan digestión lisosómica y los cuerpos autolisosómicos pueden presentar fragmentos de microvellosidades en ellos. En muchos casos, las microvellosidades pueden observarse en las membranas de células epiteliales laterales.

Las inclusiones de microvellosidades y un incremento de los gránulos secretores han sido descritos tanto en el intestino delgado como en el intestino grueso [18], pero no en el estómago; no se han observado en el epitelio renal ni en el hígado, pero aparecen en el epitelio de la vesícula biliar.

El ribete en cepillo es anormal en esta enfermedad, tal como indica el término atrofia de las microvellosidades. No obstante, debe tenerse en cuenta que en la atrofia de las microvellosidades congénita las microvellosidades son normales en la región de las criptas y se presentan variablemente acortadas o ausentes en la vellosidad. En los casos de 'inicio tardío' aparecen microvellosidades normales en la región baja de las vellosidades. En la atrofia de las microvellosidades 'atípica', las microvellosidades son cortas o están ausentes en las células superiores de las criptas y reaparecen en el epitelio expuesto en la superficie. Estas pautas de aspecto microvelloso discurren paralelamente a la distribución de gránulos secretores, es decir, tras la aparición de los gránulos las microvellosidades se acortan y, si se reduce el número de gránulos secretores, las microvellosidades reaparecen [12].

\section{Diagnóstico}

El diagnóstico puede establecerse fácilmente mediante tinción APS y ME de transmisión de biopsias de la mucosa del intestino delgado proximal. El enfoque más simple consiste en realizar la tinción APS en tejido fijado con formalina, bien orientado, e indagar la presencia de una acumulación de material APS positivo en el citoplasma apical epitelial, habitualmente en la parte superior de la cripta o en la parte inferior de la región de las vellosidades. Si se detecta dicho material debe efectuarse a continuación una ME de transmisión para determinar la posibilidad de observar inclusiones de microvellosidades. Éstas se presentarían en células epiteliales localizadas en la superficie, de manera que la orientación es importante y aparecerían dificultades en una muestra cortada transversalmente.

Se han propuesto varias técnicas de tinción como herramientas diagnósticas, entre las que destacan la fosfatasa alcalina [37], el antígeno carcinoembrionario [38] y el antígeno CD10 [39], para evitar la necesidad de realizar una ME que, aunque importante [40], se contempla como técnicamente exigente. Esas técnicas pueden demostrar la presencia de inclusiones de microvellosidades más bien que de una anomalía APS. Existe una mejor posibilidad de diagnóstico definitivo si se consideran ambas anomalías, y existe un peligro de diagnóstico erróneo si solo se utilizan medios histoquímicos, dado que la tinción positiva también puede localizarse en los autofagosomas.

\section{Patogénesis}

La patogénesis de la enfermedad es desconocida. La presencia de inclusiones de microvellosidades indica la posible existencia de un trastorno básico del ensamblaje citoesquelético. No obstante, el hallazgo de microvellosidades normales en células, en sus fases preliminares de desarrollo, en los casos 'congénitos' y de 'inicio tardío' y la reaparición de microvellosidades en los casos 'atípicos' [12] indican que esto es improbable. Las principales pro- 
teínas citoesqueléticas de las microvellosidades están presentes en el citoplasma apical de las células de las criptas, proporcionando una fuente para el crecimiento y el mantenimiento del ribete en cepillo [41]. Y este sistema puede ser perturbado dando lugar a una construcción de microvellosidades prematura dentro de la célula (fig. 1a) o a un crecimiento inapropiado en la membrana de las células laterales. Las inclusiones de microvellosidades pueden aparecer por envoltura de la membrana apical (fig. 1b) $[4,42]$. La captación de material luminal dentro de las inclusiones de microvellosidades respalda esta hipótesis [42]. Estas posibilidades no son notablemente exclusivas.

La prevalencia de gránulos secretores (fig. 2) indica una anomalía de la exocitosis del material destinado al ribete en cepillo. Las vías constitutivas, directas e indirectas, de las enzimas del ribete en cepillo permanecen intactas [43] (aunque los niveles de enzimas están reducidos debido al daño celular [12]), lo que implica una anomalía de una vía de transporte no identificada. En un trabajo preliminar se informaba de una deficiencia de miosina en el ribete en cepillo [44], lo que permite suponer una anomalía de las interacciones entre la actina y la miosina, responsable del suministro de sustancias asociadas a la membrana al ribete en cepillo [45]. Ameen y Salas [46], en un informe clínico único, también habían dejado entrever una anomalía del transporte en la membrana apical.

La naturaleza de los gránulos secretores es desconocida. Normalmente ocupan un 2,5\% del volumen de las células apicales, mientras que en la atrofia de las microvellosidades es del 40\% [46]. Datos recientes, demostrativos de la presencia de ácido siálico acetilado y antígenos de grupos sanguíneos en los gránulos secretores, indican que contienen material de tipo glucocáliz, que es bloqueado a partir de la exocitosis [47]. Esto está respaldado por la naturaleza floculenta, fibrilar, de su contenido (fig. 1a). El glucocáliz forma una capa de gel polianiónico hidrofílico sobre la superficie de las células y mantiene la carga de la superficie celular, las protege frente a traumatismos físicos y regula el acceso iónico y macromolecular [48]. Una ausencia del glucocáliz tendría consecuencias importantes sobre la función celular normal, y la acumulación de desechos celulares dentro de la célula puede perturbar los procesos normales. Dentro del útero, el intestino está bañado con líquido amniótico de osmolalidad similar a la del suero, lo que resulta en una presión no osmótica en todo el intestino. Esto cambia espectacularmente después del nacimiento, y la ausencia de glucocáliz intestinal funcional podría explicar el rápido inicio de los síntomas y el motivo por el cual la mayoría de los casos de atrofia de las microvellosidades podría ser consecuencia de la incapacidad del glucocáliz para la exocitosis [49].

\section{Enteropatía en penacho/displasia epitelial}

La enteropatía en penacho o displasia epitelial fue descrita por Reifen et al. [50] en 3 niños. Se informó de que la diarrea había tenido lugar en el periodo neonatal. Un caso preliminar fue descrito por Davidson et al. [4], subrayando la causa variable de la diarrea intratable en estos casos. La nutrición redujo espectacularmente el volumen de las deposiciones, y el apoyo nutricional permitió alcanzar una velocidad de crecimiento normal en 2 casos. La observación clave fue la presencia de enteropatía con penachos de enterocitos de superficie estrechamente compactados, con redondeamiento de la membrana apical que parecía desprenderse del epitelio. Estas características no eran únicas de este proceso y se han comunicado en la celiaquía, la atrofia de las microvellosidades, la enteropatía autoinmune y en controles, pero con una frecuencia muy inferior (90 frente a $61-<10 \%$ ) [50]. Los penachos no se observaban en el epitelio de las criptas, no existían características ultraestructurales distintivas ni incremento de la infiltración de células en la lamina propria, y la enteropatía persistía a pesar de la mejoría obtenida en 2 casos con el apoyo nutricional. Esto sitúa a este proceso en la categoría de trastorno congénito de los enterocitos en lugar de anomalía inmunológica. Se realizaron observaciones similares en 6 niños [51] con datos adicionales referentes a anomalías de la membrana basal, es decir, reducción de laminina y aumento de sulfato de heparano; sin embargo, no pudo establecerse la naturaleza primaria o secundaria de las anomalías. Patey et al. [52] investigaron las moléculas de adhesión y hallaron una mayor presencia de desmogleína, una distribución anormal de integrina $\alpha_{2} \beta_{1}$ a lo largo del eje criptavellosidad y una mayor presencia de desmosomas. Dedujeron la existencia de interacciones normales entre célula y célula y entre célula y matriz, y que dichas interacciones estaban implicadas en la patogénesis.

El proceso se considera en general como autonómico recesivo. Los padres consanguíneos y la presencia de varios hermanos afectados son corrientes [15]. Aunque Reifen et al. [50] describieron la presencia de un colon normal en sus casos, en trabajos ulteriores se demostró la afectación del colon con algunas anomalías de las criptas (ramificación y seudoquistes) [52]. El diagnóstico no es 
necesariamente sencillo, dado que la prevalencia de penachos parece variar con el tiempo y se precisan biopsias repetidas para establecer el diagnóstico, junto a una exclusión definida de la atrofia de las microvellosidades [15]. En algunos casos puede estar presente una inflamación de la lamina propria como fenómeno secundario, y el tratamiento puede permitir un aumento de la ingestión calórica [53]. Se dispone de algunas pruebas a favor de que el proceso podría resolverse en el trascurso del tiempo. Actualmente, dos pacientes adultos, después de recibir tratamiento durante 13-15 años, han podido dejar la nutrición parenteral [53], y se dispone de la descripción de un embarazo satisfactorio en una mujer con enteropatía en penacho [54].

La aclaración adicional de este proceso está a la espera de análisis genéticos moleculares. Aunque se ha descrito una relación entre la enteropatía en penacho y las polimalformaciones [55], puede tratarse de una coincidencia más bien que de un vínculo genético.

\section{Deficiencia de integrinas}

Lachaux et al. [56] describieron recientemente el caso de un lactante con atresia pilórica y diarrea intratable. La microscopía óptica mostró una extensa descamación grave de células epiteliales, desde el estómago hasta el recto, permaneciendo únicamente en su lugar las células epiteliales de las criptas. La ME intestinal reveló la presencia de desmosomas normales con separación entre la lamina propria y la membrana basal de los enterocitos. Este trastorno se considera relacionado con una deficiencia congénita de integrina $\alpha_{6} \beta_{4}$, pero de una isoforma intestinal, dado que la expresión cutánea de la integrina $\alpha_{6} \beta_{4}$ parecía normal. Esta integrina es defectuosa en la epidermolisis bullosa, en la cual se produce un macrodesprendimiento epidérmico. Es probable que se trate de una mutación dentro de la isoforma $\alpha_{6} \beta_{4}$ intestinal o una deficiencia de una integrina intestinal relacionada, con reacción cruzada en términos inmunohistoquímicos.

\section{Diarrea sindromática}

Se ha descrito un reducido número de pacientes con diarrea intratable sindromática [57, 58]. Anteriormente se habían comunicado casos similares que correspondían al síndrome 'trico-hepato-entérico' [59-61]. En este síndrome, los casos son pequeños para la edad gestacional y presentan diarrea intratable que se inicia en los primeros meses de vida, insuficiencia hepática, dismorfismo facial, retraso mental y pelo anormal. La morfología del intestino delgado es variablemente anormal, entre una atrofia de las vellosidades grave y un acortamiento menor de las vellosidades. También se ha descrito colitis [57]. Los padres consanguíneos son corrientes [58] y se han descrito hermanos afectos del síndrome [59,61], lo que permite suponer un proceso autosómico recesivo. Algunos casos presentan signos de inmunodeficiencia con respuestas pobres a la vacunación e hipogammaglobulinemia.

El pronóstico es variable; algunos casos han sucumbido a la infección o a la insuficiencia hepática, otros requieren nutrición parenteral total o parcial y uno de los casos está recibiendo nutrición oral [58]. Se ha dado a entender que la tolerancia a los alimentos enterales estaba relacionada con el estado del intestino delgado [58]. La relación entre la diarrea intratable sindromática y el síndrome 'trico-hepato-entérico' tiene que ser evaluada adicionalmente. El cuadro clínico sugiere una mutación de varios genes, heredada conjuntamente por desequilibrio de acoplamiento, o interferencia con un mayor nivel de control, como un gen modelado; dado que son relativamente pocos los genes modelados que participan en el desarrollo del pelo, este hecho puede permitir una búsqueda más selectiva de mutaciones candidatas [15].

\section{Trastornos congénitos de la glucosilación y enteropatía con pérdida de proteínas}

La diarrea se ha asociado variablemente a trastornos congénitos de la glucosilación, y en un trabajo se describe una diarrea intratable grave a las 2 semanas de edad como síntoma persistente en condiciones de nutrición parenteral, acompañado de una acusada hipoalbuminemia [62]. Aparecieron síntomas neurológicos, no se obtuvo ninguna respuesta al tratamiento con manosas y el paciente falleció a los 24 meses de edad por insuficiencia hepática. La anomalía de glucosilación básica, más allá de una anomalía de tipo 1 , no ha podido identificarse.

Se comunicaron tres casos similares con diarrea intratable y enteropatía con pérdida de proteínas grave [63]. En estos casos, aunque el aspecto de la mucosa del intestino delgado era histológicamente normal, se apreciaba una ausencia de sulfato de heparano en las membranas de las células epiteliales laterales. Recientemente, se ha establecido experimentalmente un vínculo entre sulfato de heparano, enteropatía con pérdida de proteínas e inflamación [64]. Todos requirieron nutrición parenteral total, si bien la diarrea persistió. Aunque no se describie- 
ron anomalías neurológicas ni otros signos del síndrome de anomalía de la glucosilación, se comunicó un caso de trastorno congénito de la glucosilación de tipo 1c con hipoalbuminemia grave y una ausencia similar de sulfato de heparano en el epitelio intestinal [65]; por otra parte, los pacientes con trastornos congénitos de la glucosilación de tipo $1 \mathrm{~b}$ presentaban diarrea grave y enteropatía con pérdida de proteínas [66], por lo cual pueden existir similitudes entre los diversos procesos.

\section{Otras causas y resumen}

A pesar de la investigación intensiva de los casos de diarrea intratable, siguen habiendo numerosos casos en los que la búsqueda de signos diagnósticos característicos es esquiva. Se espera que a medida que se descubra la base genética de enfermedades con características distintivas, podrán aclararse aspectos de estos procesos idiopáticos. Sin duda, la dedicación de los equipos de gastroenterología pediátrica que asisten a estos niños con trastornos del desarrollo de los enterocitos está facilitando el tratamiento y el establecimiento de diagnósticos separados, que permiten una investigación selectiva y productiva. No obstante, las diarreas y enteropatías causadas por enfermedades congénitas basadas en anomalías de los enterocitos persisten frecuentemente a pesar de las intervenciones médicas, a menos que se efectúe un trasplante intestinal. Existe la seguridad de que las posibilidades de resolución de estas enfermedades graves y exigentes deben esperar hasta poder disponer de tratamientos génicos satisfactorios.

\section{Bibliografía}

$D_{1}$ Avery GB, Villavicencio O, Lilly JR, Randolph 11 Cutz E, Rhoads JM, Drumm B, Sherman PM, JG: Intractable diarrhoea in early infancy. Pediatrics 1968;41:712-722.

-2 Larcher VF, Shepherd R, Francis DEM, Harries JT: Protracted diarrhoea in infancy. Analysis of 82 cases with particular reference to diagnosis and management. Arch Dis Child 1977;52:597-605.

3 Hyman CJ, Reiter J, Rodnan J, Drash AL: Parenteral and oral alimentation in the treatment of the nonspecific protracted diarrheal syndrome of infancy. J Pediatr 1971;78:17-29.

-4 Davidson GP, Cutz E, Hamilton JR, Gall DG: Familial enteropathy: a syndrome of protracted diarrhea from birth, failure to thrive, and hypoplastic villous atrophy. Gastroenterology 1978;75:783-790

-5 Candy DCA, Larcher VF, Cameron DJS, et al: Lethal familial protracted diarrhoea. Arch Dis Child 1981;56:15-23.

6 Cuenod B, Brousse N, Goulet O, et al: Classification of intractable diarrhea in infancy using clinical and immunohistological criteria. Gastroenterology 1990;99:1037-1043.

-7 Goulet OJ, Brousse N, Canioni D, WalkerSmith JA, Schmitz J, Phillips AD: Syndrome of intractable diarrhoea with persistent villous atrophy in early childhood: a clinicopathological survey of 47 cases. J Pediatr Gastroenterol Nutr 1998;26:151-161.

8 Goulet O, Ruemmele F, Lacaille F, Colomb V: Irreversible intestinal failure. J Pediatr Gastroenterol Nutr 2004;38:250-269.

${ }_{9}$ Sanderson IR, Risdon RA, Walker-Smith JA: Intractable ulcerating enterocolitis of infancy. Arch Dis Child 1991;66:295-299.

10 Thapar N, Shah N, Ramsay AD, Lindley KJ, Milla PJ: Long-term outcome of intractable ulcerating enterocolitis of infancy. J Pediatr Gastroenterol Nutr 2005;40:582-588.
Durie PR, Forstner GG: Microvillus inclusion disease: an inherited defect of brush-border assembly and differentiation. $\mathrm{N}$ Engl $\mathrm{J}$ Med 1989;320:646-651.

12 Phillips AD, Schmitz J: Familial microvillous atrophy: a clinicopathological survey of 23 cases. J Pediatr Gastroenterol Nutr 1992;14: 380-396.

13 Cutz E, Sherman PM, Davidson GP: Enteropathies associated with protracted diarrhea of infancy: clinicopathological features, cellular and molecular mechanisms. Pediatr Pathol Lab Med 1997; 17:335-368.

14 Sherman PM, Mitchell DJ, Cutz E: Neonatal enteropathies: defining the causes of protracted diarrhea of infancy. J Pediatr Gastroenterol Nutr 2004;38:16-26.

15 Goulet O, Phillips AD: Congenital enteropathy involving intestinal mucosa development; in Walker WA, Goulet O, Kleinman RE, Sanderson IR, Sherman P, Shneider B (eds): Paediatric Gastrointestinal Disease. Burlington, Decker, 2004, pp 922-931.

16 Schmitz J, Ginies JL, Arnaud-Battandier F, et al: Congenital microvillous atrophy: a rare cause of neonatal intractable diarrhoea. Pediatr Res 1982;16:1014.

17 Goutet JM, Boccon-Gibod L, Chatelet F, Ploussard JP, Navarro J, Polonovski Cl: Familial protracted diarrhoea with hypoplastic villous atrophy: report of two cases. Pediatr Res 1982;16:1045.

18 Phillips AD, Jenkins P, Raafat F, WalkerSmith JA: Congenital microvillous atrophy: specific diagnostic features. Arch Dis Child 1985;60:135-140.
19 Beck NS, Chang YS, Kang IS, Park WS, Lee HJ, Suh YL: Microvillus inclusion disease in two Korean infants. J Korean Med Sci 1997; 12:452-456.

20 Kaneko K, Shimizu T, Fujiwara S, Igarashi J, Ohtomo Y, Yamashiro Y: Microvillous inclusion disease in Japan. J Pediatr 1999;135: 400 .

21 Pohl JF, Shub MD, Trevelline EE, et al: A cluster of microvillous inclusion disease in the $\mathrm{Na}$ vajo population. J Pediatr 1999;134:103106.

22 Kennea N, Norbury R, Anderson G, Tekay A: Congenital microvillous inclusion disease presenting as antenatal bowel obstruction. Ultrasound Obstet Gynecol 2001;17:172-174.

23 Makela S, Kere J, Holmberg C, Hoglund P: SLC26A3 mutations in congenital chloride diarrhea. Hum Mutat 2002;20:425-438.

24 Keller KM, Wirth S, Baumann W, Sule D, Booth IW: Defective jejunal brush border membrane sodium/proton exchange in association with lethal familial protracted diarrhoea. Gut 1990;31:1156-1158.

25 Croft NM, Howatson AG, Ling SC, Nairn L, Evans TJ, Weaver LT: Microvillous inclusion disease: an evolving condition. J Pediatr Gastroenterol Nutr 2000;31:185-189.

26 Ruemmele FM, Jan D, Lacaille F, et al: New perspectives for children with microvillous inclusion disease: early small bowel transplantation. Transplantation 2004;77:1024-1028.

27 Randak C, Langnas AN, Kaufman SS, et al: Pretransplant management and small bowelliver transplantation in an infant with microvillus inclusion disease. J Pediatr Gastroenterol Nutr 1998;27:333-337.

28 Nathavitharana KA, Green NJ, Raafat F, Booth IW: Siblings with microvillous inclusion disease. Arch Dis Child 1994;71:71-73. 
-29 Assmann B, Hoffmann GF, Wagner L, et al: Dihydropyrimidinase deficiency and congenital microvillous atrophy: coincidence or genetic relation? J Inherit Metab Dis 1997;20:681688.

30 Heinz-Erian P, Schmidt H, Le Merrer M, Phillips AD, Kiess W, Hadorn HB: Congenital microvillus atrophy in a girl with autosomal dominant hypochondroplasia. J Pediatr Gastroenterol Nutr 1999;28:203-205.

- 31 Raafat F, Green NJ, Nathavitharana KA, Booth IW: Intestinal microvillous dystrophy: a variant of microvillous inclusion disease or a new entity? Hum Pathol 1994;25:1243-1248.

-32 Mierau GW, Wills EJ, Wyatt-Ashmead J, Hoffenberg EJ, Cutz E: Microvillous inclusion disease: report of a case with atypical features. Ultrastruct Pathol 2001;25:517-521.

33 Weeks DA, Zuppan CW, Malott RL, Mierau GW: Microvillous inclusion disease with abundant vermiform, electron-lucent vesicles. U1trastruct Pathol 2003;27:337-340.

- 34 Walker-Smith JA, Phillips AD, Walford N, et al: Intravenous epidermal growth factor/urogastrone increases small intestinal cell proliferation in congenital microvillous atrophy. Lancet 1985;ii:1239-1240.

-35 Groisman GM, Sabo E, Meir A, Polak-Charcon S: Enterocyte apoptosis and proliferation are increased in microvillous inclusion disease (familial microvillous atrophy). Hum Pathol 2000;31:1404-1410.

- 36 Phillips AD, Szafranski M, Man LY, Wall WJ: Periodic acid-Schiff staining abnormality in microvillous atrophy: photometric and ultrastructural studies. J Pediatr Gastroenterol Nutr 2000;30:34-42.

-37 Lake BD: Microvillus inclusion disease: specific diagnostic features shown by alkaline phosphatase histochemistry. J Clin Pathol 1988;41:880-882.

- 38 Groisman GM, Ben-Izhak O, Schwersenz A, Berant M, Fyffe B: The value of polyclonal carcinoembryonic antigen immunostaining in the diagnosis of microvillus inclusion disease. Hum Pathol 1993;24:1232-1237.

- 39 Groisman GM, Amar M, Livne E: CD10: a valuable tool for the light microscopic diagnosis of microvillous inclusion disease (familial microvillous atrophy). Am J Surg Pathol 2002; 26:902-907.

-40 Bell SW, Kerner JA, Sibley RK: Microvillous inclusion disease. The importance of electron microscopy for diagnosis. Am J Surg Pathol 1991;15:1157-1164.

-41 Fath KR, Obenauf SD, Burgess DR: Cytoskeletal protein and mRNA accumulation during brush border formation in adult chicken enterocytes. Development 1990;109:449-459.
42 Reinshagen K, Naim HY, Zimmer KP: Autophagocytosis of the apical membrane in microvillus inclusion disease. Gut 2002;51:514521.

-43 Phillips AD, Fransen JAM, Hauri HP, Sterchi E: The constitutive exocytotic pathway in microvillous atrophy. J Pediatr Gastroenterol Nutr 1993; 17:239-246.

44 Carruthers L, Phillips AD, Dourmashkin R, Walker-Smith JA: Biochemical abnormality in brush border membrane protein of a patient with congenital microvillous atrophy. J Pediatr Gastroenterol Nutr 1985;4:902-907.

45 Fath KR, Trimbur GM, Burgess DR: Molecular motors are differentially distributed on Golgi membranes from polarized epithelial cells. J Cell Biol 1994;126:661-675.

46 Ameen NA, Salas PJ: Microvillus inclusion disease: a genetic defect affecting apical membrane protein traffic in intestinal epithelium. Traffic 2000;1:76-83.

47 Phillips AD, Brown A, Hicks S, et al: Acetylated sialic acid residues and blood group antigens localise within the epithelium in microvillous atrophy indicating internal accumulation of the glycocalyx. Gut 2004;53:1764-1771.

48 Gupta BL: The relationship of mucoid substances and ion and water transport, with new data on intestinal goblet cells and a model for gastric secretion. Symp Soc Exp Biol 1989;43: 81-110.

49 Gillibrand PN: Changes in the electrolytes, urea and osmolality of the amniotic fluid with advancing pregnancy. J Obstet Gynaecol $\mathrm{Br}$ Commonw 1969;76:898-905.

50 Reifen RM, Cutz E, Griffiths AM, Ngan BY, Sherman PM: Tufting enteropathy: a newly recognized clinicopathological entity associated with refractory diarrhea in infants. J Pediatr Gastroenterol Nutr 1994;18:379-385.

-51 Goulet O, Kedinger M, Brousse N, et al: Intractable diarrhea of infancy with epithelial and basement membrane abnormalities. J Pediatr 1995; 127:212-219.

52 Patey N, Scoazec JY, Cuenod-Jabri B, et al: Distribution of cell adhesion molecules in infants with intestinal epithelial dysplasia (tufting enteropathy). Gastroenterology 1997;113: 833-843.

53 Schaeppi M, Shah NF, Long S, et al: Tufting enteropathy also affects the colon. J Pediatr Gastroenterol Nutr 1999;28:569.

54 Cameron DJ, Barnes GL: Successful pregnancy outcome in tufting enteropathy. J Pediatr Gastroenterol Nutr 2003;36:158.

55 Abely M, Hankard GF, Hugot JP, Cezard JP, Peuchmaur M, Navarro J: Intractable infant diarrhea with epithelial dysplasia associated with polymalformation. J Pediatr Gastroenterol Nutr 1998;27:348-352.
56 Lachaux A, Bouvier R, Loras-Duclaux I, Chappuis JP, Meneguzzi G, Ortonne JP: Isolated deficient alpha6beta4 integrin expression in the gut associated with intractable diarrhea. $\mathrm{J}$ Pediatr Gastroenterol Nutr 1999;29:395401.

57 Girault D, Goulet O, Le Deist F, et al: Intractable infant diarrhea associated with phenotypic abnormalities and immunodeficiency. J Pediatr 1994;125:36-42.

58 Martinez-Vinson C, Goulet O, Berrebi D, et al: Syndromatic diarrhea in children. Report of 8 cases. J Pediatr Gastroenterol Nutr 2005;40: 651.

59 Verloes A, Lombet J, Lambert Y, et al: Trichohepato-enteric syndrome: further delineation of a distinct syndrome with neonatal hemochromatosis phenotype, intractable diarrhea, and hair anomalies. Am J Med Genet 1997;68: 391-395.

-60 Landers MC, Schroeder TM: Intractable diarrhea of infancy with facial dysmorphism, trichorrhexis nodosa, and cirrhosis. Pediatr Dermatol 2003;20:432-435.

61 Stankler L, Lloyd D, Pollitt RJ, Gray ES, Thom H, Russell G: Unexplained diarrhoea and failure to thrive in 2 siblings with unusual facies and abnormal scalp hair shafts: a new syndrome. Arch Dis Child 1982;57:212-216.

62 Mention K, Michaud L, Dobbelaere D, Guimber D, Gottrand F, Turck D: Neonatal severe intractable diarrhoea as the presenting manifestation of an unclassified congenital disorder of glycosylation (CDG-x). Arch Dis Child Fetal Neonatal Ed 2001;85:F217-F219.

63 Murch SH, Winyard PJ, Koletzko S, et al: Congenital enterocyte heparan sulphate deficiency with massive albumin loss, secretory diarrhoea, and malnutrition. Lancet 1996;347: 1299-1301.

64 Bode L, Eklund EA, Murch S, Freeze HH Heparan sulfate depletion amplifies TNF-alpha-induced protein leakage in an in vitro model of protein-losing enteropathy. Am J Physiol Gastrointest Liver Physiol 2005;288: G1015-G1023.

65 Westphal V, Murch S, Kim S, et al: Reduced heparan sulfate accumulation in enterocytes contributes to protein-losing enteropathy in a congenital disorder of glycosylation. Am J Pathol 2000;157:1917-1925.

-66 Niehues R, Hasilik M, Alton G, et al: Carbohydrate-deficient glycoprotein syndrome type Ib. Phosphomannose isomerase deficiency and mannose therapy. J Clin Invest 1998;101: $1414-1420$ 